\title{
TEOREMA DE PITÁGORAS PARA ESTUDANTES DA PEDAGOGIA NA UNIVERSIDADE FEDERAL DO CEARÁ: FOCO NA MEDIAÇÃO DO ENSINO
}

\section{PYTHAGOREAN THEOREM FOR PEDAGOGY STUDENTS IN FEDERAL UNIVERSITY OF CEARÁ: FOCUS ON EDUCATION MEDIATION}

MAGALHÃES ${ }^{1}$, Elisângela Bezerra; BRANDÃO, Jorge Carvalho; SANTOS, Maria José Costa dos.

Universidade Federal do Ceará

Recebido em: 12/08/2017; Aceito: 06/11/2017; Publicado: 01/12/2017

\begin{abstract}
RESUMO
Pretende-se por meio desse trabalho apresentar as reflexões e socializar os resultados obtidos durante a pesquisa feita com acadêmicos do curso de pedagogia da Universidade Federal do Ceará (UFC) na disciplina tópicos de educação matemática, considerando que os conhecimentos matemáticos são essencial a sociedade atual, observar a respeito da matemática e se justifica mediante a compreensão das dificuldades que frequentemente permeiam o processo de ensino aprendizagem da matemática, ou de disciplinas a ela relacionadas. Esse estudo é parte de uma proposta desenvolvida coletivamente por pesquisadores do GEM (Grupo do Ensino da matemática), visando identificar os sentidos da matemática nos diversos contextos sociais e, a partir da representação identificada pontuar questões relevantes à educação, dentre elas a utilização de uma metodologia específica para o ensino da matemática. A proposta da pesquisa foi trabalhar de forma mediada os capítulos do livro o Homem que calculava de Malba Tahan resolvendo as questões e problemas apresentados no livro subsidiado por metodologias de mediação. O presente estudo foi desenvolvido por uma estudante de doutorado, e dois professores da referida Universidade, e aplicada para apresentação de estudantes da turma de pedagogia.

Palavras chaves: ensino da matemática, mediação, Malba Tahan.
\end{abstract}

\section{SUMMARY}

It is intended through this work present the reflections and socialize the results obtained during the survey of students of the pedagogy course at the Federal University of Ceará (UFC) in the course topics of mathematics education, considering that mathematical knowledge is essential in today's society observe about the mathematics and is justified by understanding the difficulties that often permeate the process of teaching and learning of mathematics, or subjects related to it. This study is part of a proposal collectively developed by researchers at the GEM (Mathematics Education Group), to identify the meanings of mathematics in different social contexts, and from the identified representation scoring issues relevant to education, among them the use of a specific methodology for teaching mathematics. The purpose of the research was work mediated form the chapters of the book the man who calculated Malba Tahan solving issues and problems presented in the book subsidized by methodologies of mediation. This study was developed by a doctoral student and two teachers of the EHU, and applied to present students of pedagogy class.
Key
words:
mathematics
teaching,
mediation,
Malba
Tahan.

\footnotetext{
1 Doutoranda em Educação Brasileira, Mestre em Educação Brasileira pela UFC, psicopedagoga Clinica e Institucional. Pesquisadora em educação matemática adaptada para deficientes visuais. Tem experiência na área de Matemática, com ênfase em Matemática Inclusiva, atuando principalmente nos seguintes temas: Matemática adaptada para pessoas com dificuldades de aprendizagem. Adaptação de material para ensino de matemática para deficientes visuais. Metodologias de ensino para deficientes visuais. E-mail: lala2magalhaes@gmail.com telefone: (85) 987889869
} 


\section{INTRODUÇÃO}

Durante a disciplina Tópicos de Educação Matemática que é ofertada para os alunos do curso de Pedagogia da Universidade Federal do Ceará (UFC), desenvolveu-se o locos dessa pesquisa, durante as aulas incidiu as observações e a utilização da metodologia de mediação de ensino, o objetivo foi trabalhar o conceito do Teorema de Pitágoras, sugerido no capitulo 18 do livro Malba Tahan intitulado: Uma reunião dos poetas e letrados. A homenagem ao Marajá de Leore. A matemática na Índia. A pérola de Lilavati. O problema de Aritmética dos hindus. O valor da escrava de 20 anos. Nesse capítulo é apresentado um problema que sua resposta está em entender o Teorema de Pitágoras.

Nossa proposta então foi de trabalhar esse conceito através da metodologia de mediação de ensino utilizando material concreto e jogos. Já que os estudantes trazem o discurso de dificuldade de aprender matemática.

O Teorema de Pitágoras é considerado um dos mais importantes na geometria euclidiana. Já foi demonstrado por várias civilizações e de várias formas no decorrer da história. Nessa perspectiva entendemos que é de extrema importância que o professor seja ele pedagogo ou licenciado ao ensinar matemática tenha conhecimento da história, ao trabalhar este conteúdo com seus alunos. No entanto observamos que ensinar matemática é um grande desafio ao professor, muitos motivos estão envolvidos nesse processo, o desinteresse dos estudantes, por conta que na atualidade o uso da tecnologia, não proporciona que os estudantes desenvolvam o ato de investigar, pesquisar e estudar, preferindo sempre o que já esta pronto. Outro obstáculo no ensino da matemática é o não conhecimento como o desinteresse por parte dos professores em conhecerem e entenderem a da matemática e acabam deixando-a de lado.

Analisando esse quadro, durante a disciplina de tópicos em educação matemática, propomos o trabalho desses conceitos, na pedagogia através de metodologias de mediação de ensino.

De acordo com Libâneo (1994) o ensino tem como função principal garantir o processo de transmissão e assimilação dos conteúdos do saber escolar, através desse processo, o desenvolvimento das capacidades cognoscitivas dos alunos, de maneira que, o professor planeje, dirija e comande o processo de ensino, tendo em vista estimular e suscitar a atividade própria dos alunos para a aprendizagem. Corroborando com o autor BRANDÃO (2014) enfatiza que "durante muito tempo confundiu-se "ensinar" com "transmitir" e nesse contexto o aluno era um agente passivo da aprendizagem e o professor um simples transmissor nem sempre presente na necessidade dos alunos".

Nessa perspectiva desenvolvemos aulas na pedagogia pensando no ensino como uma atividade que vá além do ato de se estabelecer uma ação colaborativa entre docente e discente, necessitando essa ação ser aberta e socializada com os demais estudantes, onde todos troquem conhecimentos e discutam sendo possível organizar conceitos, sem haja a necessidade de modelos e esquemas prontos. Assim, ensinar é possibilitar que estudantes produzam e elaborem conhecimento, de modo que possam sistematizar a informação e expressar tanto suas 
dúvidas e incertezas. Nesse sentido, oferecer ao estudante de pedagogia a oportunidade de construir uma prática docente baseada em métodos de mediação faz-se necessário com intuito de que cada um comece a transformar sua práxis.

Desse modo, percebemos a importância de favorecer aos estudantes de pedagogia o conhecimento de metodologias que mediam a ação docente.

O professor deve se colocar como ponte entre o estudante e o conhecimento para que, dessa forma, o aluno aprenda a "pensar" e a questionar por si mesmo e não mais receba passivamente as informações como se fosse um depósito do educador. (BULGRAEN, 2010, p. 31).

Nesse trabalho apresentaremos como foi feita a mediação dos conteúdos ministrados na disciplina Tópicos em educação matemática, através da metodologia Sequência Fedathi, a metodologia está aportada no trabalho do professor mediador, preocupa-se com a postura e atitude da ação docente. $\mathrm{O}$ conceito do Teorema de Pitágoras será mediado através das fases da referida metodologia e desenvolvida através da utilização de recursos adaptados e construídos com material de baixo custo, afim de que os estudantes de pedagogia percebam a importância da elaboração do conceito pelo aluno de forma significativa.

\section{SEQUÊNCIA FEDATHI E TEOREMA DE PITÁGORAS}

Piaget (1987); Vygotsky (2002); Rogers (1985); Wallom (1973), entre outros, são teóricos que pesquisaram sobre a teoria de aprendizagem de cujo objeto de estudo está voltada ao tipo de aprendizagem desenvolvido pelos alunos. Magalhães (2015) afirma que os autores defendem a ideia de que conhecer as condições cognitivas do aluno, seus processos mentais, possibilita constituir e interpretar a elaboração do conhecimento que chamamos de aprendizagem.

Para esse estudo ao utilizarmos a metodologia iremos observar a importância de se trabalhar o ensino baseado na postura do professor ao ministrar conteúdos. A Sequência Fedathi visa oferecer ao docente a oportunidade de trabalhar significativamente o aluno, a fim de favorecer um momento de aprendizagem onde ele possa utilizar para toda sua vida e tenha condição de fazer parte ativa desse processo. Já que o principal objetivo de se mediar o ensino se baseia na promoção da maior autonomia e elaboração dos conhecimentos pelos estudantes, favorecendo que os mesmos decidam e sejam capazes de solucionar problemas. A metodologia FEDATHI "busca diferenciar-se positivamente em relação ao ensino tradicional, valorizando igualmente as ações do professor e do aluno durante o ensino". (SOUZA 2013, p.38)

Para ministrar conteúdos utilizando a Sequência Fedathi é sugerido que o professor faça a mediação do ensino através de quatro etapas: tomada de posição. Maturação, solução e prova.

$\mathrm{Na}$ tomada de posição o professor apresenta uma situação desafiadora ao grupo, estabelece um contrato de como a aula vai se desenvolver e deixa claro que o aluno faz parte ativa do processo de ensino. Maturação momento onde ambas as partes docentes e discentes dão inicio as discussões sobre a 
resolução do problema apresentado. Na solução os discentes têm a oportunidade de organizar o que encontraram de solução e apresentar para o grupo para se desenvolver o debate sobre como se chegou àquela solução. E a prova $\mathrm{O}$ professor demonstra o novo conhecimento (SOUZA 2013). O professor avalia as considerações dos alunos, e apresenta a solução do problema.

$$
\text { Considerando as etapas citadas }
$$
apresentaremos todo o processo aplicado à turma de pedagogia no conceito do teorema de Pitágoras durante as aulas da disciplina.

\section{AÇÕES MEDIADAS PELA SEQUÊNCIA FEDATHI}

Como já mencionado, a ação do estudo foi desenvolvida em uma aula do curso de pedagogia, com foco na metodologia Sequência Fedathi, envolvendo materiais concretos. As atividades relacionadas aos materiais satisfazem os princípios educacionais apresentados nos Parâmetros Curriculares Nacionais para o ensino da geometria nas séries do ensino fundamental (BRASIL, 1998) e foram estabelecidas segundo o modelo de Van Hiele do desenvolvimento do pensamento geométrico (VAN HIELE, 1986). Os recursos didáticos e a metodologia envolvida no desenvolvimento das atividades para sala de aula permitem motivar o educando para a aprendizagem das formas e conceitos geométricos elementares, por meio da utilização de quebra-cabeças especiais e aparelhos didáticos de baixo custo.

As atividades propostas e desenvolvidas envolveram diversos três tipos de material concreto, trabalhamos com Tangram, dobradura, e com peças de E.V.A. Esses recursos foram desenvolvidos segundo várias indicações encontradas em Lorenzato (2006).
Para a confecção dos jogos e recursos concretos foram utilizados materiais de baixo custo, como papel-cartão e texturas diversas, emborrachados tipos e texturas diferentes.

As atividades sugeridas para o desenvolvimento do trabalho foram desenvolvidas concomitantemente entre os estudantes que estavam subdivido em três equipes.

\section{A primeira atividade- atividade com quadrados em EVA}

Formamos uma equipe de cinco estudantes e distribuímos os quadradinhos em EVA com duas cores o objetivo era solucionar o problema proposto em relação a solucionar o teorema de Pitágoras utilizando o tangran.

Tomada de Posição: Sugerimos que a equipe elaborasse o teorema sem olhar para modelos e construísse a partir das peças distribuídas. Os estudantes tiveram um tempo para tentarem solucionar o problema proposto.

Maturação: Durante a aula os componentes da equipe tiveram a oportunidade de discutir várias soluções e ouvir de todas as sugestões de solução do problema. Fizeram tentativas, discutiram possíveis soluções.

Nessa etapa os estudantes fizeram desenhos com as peças do tangran, discutiram os acertos e erros que aconteceu durante o desenvolvimento. Importante observar que nessa fase a Sequência Fedathi sugere que fase o professor faça intervenção de mediação proporcionando aos estudantes formular novas hipóteses de resposta.

\section{Ilustração 01 - Alunos trabalhando com as peças de EVA}




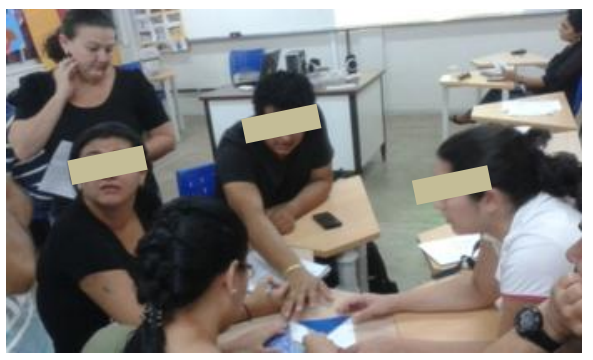

Fonte: Acervo dos autores

O momento da Solução nesse período os componentes da equipe tiveram um tempo razoável para discutirem e demonstraram entre eles quais as possibilidades para solução do problema proposto no início da aula.

A equipe tentou de vários modos solucionar o problema proposto, alguns membros da equipe apresentaram algumas dificuldades onde uma dessas dificuldades pode ser observada em relação a entender como utilizar os quadrados para elaborar as formas desejadas. No início eles tentaram pela cor, depois contando as peças e igualando as quantidades. Mas uma das dificuldades mais claras foi a definição do cateto e da hipotenusa.

Alguns comentários foram percebidos notoriamente em relação a importância da matemática. $\mathrm{O}$ que foi comentado pelos componentes da equipe é que a dificuldade encontrada foi exatamente a explicação confusa do facilitador. Embora o facilitador estivesse o tempo todo em contato com as equipes fornecendo ajuda para o desenvolvimento da atividade, os componentes não entendiam o objetivo da atividade nem o que era para ser construído e elaborado com os quadradinhos.

\footnotetext{
A matemática é às vezes tentativas, procurar tentar várias vezes até
}

achar a solução. Aluno equipe 01.

A Prova: a prova foi desenvolvida a partir de apresentação da equipe para o restante da sala e assim a demonstração dos passos que utilizaram para construção do teorema. Nesse instante o facilitador demonstrou em um vídeo como solucionar a questão e comparou com o apresentado pela equipe, e se os mesmos seguiram os passos corretos.

"No momento coletivo, a ação da professora procura conduzir à análise, pois, por meio dela, os alunos identificam em suas ações os elementos substanciais do problema" (SFORNI, 2004, p. 147). Nessa última fase da sequência, os professores formalizaram as reflexões dos estudantes sobre os conceitos e procuraram despertar nos estudantes a observação de apreciação sobre suas respostas, fazendo-os compreender a importância de suas argumentações e de suas elaborações.

\section{A Segunda atividade - Tangram adaptado}

Durante a aula sobre os conteúdos foi desenvolvida algumas indagações, sobre o tangran, para que utilizamos? Quais conteúdos podemos trabalhar com esse recurso? E quem sabe montar um Tangran! Os estudantes logo ficaram apreensivos, pois alguns não conheciam o material, e diziam que não iam conseguir cumprir a atividade.

Para a tomada de posição foi sugerido que os estudantes a partir do Tangram adaptado elaborassem o teorema de Pitágoras sem que precisasse de peças a mais do que as sete oferecidas. 
Optamos por trabalhar com peças adaptadas com materiais e texturas diferentes, pensando na possibilidade de que esse material pudesse ser utilizado por pessoas com NEE (Necessidades de Educativas Especiais) já que essas se fazem presentes em nossas salas de aula, e torna-se imprescindível que os nossos estudantes de Pedagogia, futuros professores tenham conhecimento de como trabalhar e adaptar material para esses estudantes.

\section{Ilustração 02 - estudantes trabalhando com}

Tangram adaptado

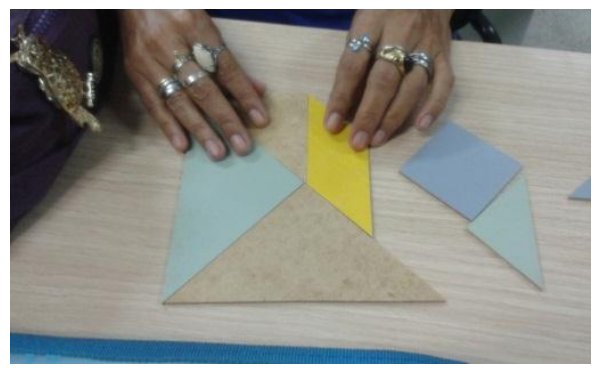

Fonte: Acervo dos autores

Durante o momento da maturação os estudantes tiveram tempo de avaliar o Tangram adaptado e sugerir soluções para o problema.

A solução foi o momento onde todos os estudantes tiveram a oportunidade de testar várias as situações de elaboração, no entanto a observação feita pelos facilitadores foi a de que os estudantes não entendiam a definição do cateto e da hipotenusa a partir do Tangram. Apresentaram dificuldades então o facilitador explicou novamente e a partir desse momento perceberam o tamanho das peças e conseguiram montar. Inicialmente construíram a hipotenusa e depois conseguiram montar o cateto.

O momento da prova foi realizado a partir das discussões em sala de aula, e apresentação do vídeo pelo apresentador sobre a solução do problema e observação dos caminhos a serem utilizados para resolução estavam corretos.

\section{A terceira atividade - dobraduras}

A equipe formada por três estudantes recebeu os papéis com dobraduras para realizar o desenho do teorema de Pitágoras, que foi a tomada de posição todos os componentes da equipe participaram da atividade que foi percebida intensidade e vontade de realizar a solução.

\section{Ilustração 03 estudantes realizando a atividade}

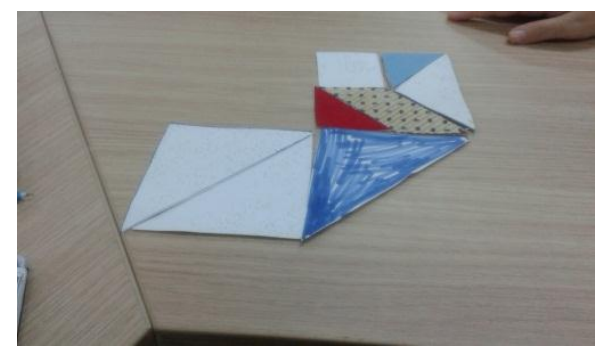

Fonte: Acervo dos autores

A maturação foi realizada a partir da tentativa dos estudantes em desenhar o teorema de Pitágoras no papel com dobraduras.

A solução foi desenvolvida a partir das discussões dos estudantes a partir das tentativas em solucionar o problema e cada um demonstrou como faria para chegar à solução. Nessa etapa apresentamos um vídeo sobre o teorema, seu surgimento, suas funções o que causou muito interesse por parte dos estudantes.

Nunca tinha pensado que poderíamos trabalhar esse teorema em sala utilizando um vídeo tão interessante.

(estudante da pedagogia)

Captamos a essência só não conseguimos representar (estudante da equipe) 
A prova consistiu no debate sugerido pelo facilitador sobre os caminhos utilizados para representar o teorema e a apresentação do conceito através do vídeo.

Ao oportunizarmos o debate em sala, os estudantes relataram a importância de se perceber que os conceitos matemáticos podem ser utilizados de forma mediada para que o estudante não desenvolva os conceitos através apenas de modelos e fórmulas prontas, observaram que o aprendizado torna-se mais significativo através de experiências concretas.

\section{RESULTADOS E DISCUSSÕES}

A pesquisa realizada apresentou um estudo relevante para o ensino da matemática básica voltada para pedagogos em formação. Isso por assumir uma postura não tradicionalista, já que a proposta da disciplina foi desenvolver várias sessões didáticas de forma lúdica, podendo se usar desde materiais adaptados, jogos educativos a manifestações artística. Essa postura torna-se positiva no tocante ao que a educação, principalmente a básica, clama nas salas de aula por uma prática mais significativa. O cenário mostra que os alunos necessitam de movimento e que a aula expositiva em que os alunos ficam sentados somente esperando o professor repassar os conhecimentos, já não são válidos.

Como se percebe essa mudança de paradigma de ação docente centrada no repasse de conteúdos para uma ação mediada, não é uma tarefa fácil, entendemos que para que haja essa transformação existe a necessidade de se trabalhar com os novos estudantes da pedagogia, para que suas ações sejam voltadas a mediação do conhecimento e não no repasse de conteúdos.

Entendemos que essas mudanças se fazem em longo prazo e que o professor deve ser consciente do seu papel na escola. Ser professor, ser professora é um privilégio. É cuidar da humanização e da dignidade das pessoas. LIBÂNEO (2001)

Por fim vamos aqui chamar a atenção da necessidade para utilização de metodologias que favoreça a mediação e que valorize a aprendizagem do estudante. Também dar mais atenção as questões da utilização dos materiais adaptados em sala de aula, pois necessitados remanejar para dentro do cotidiano escolar a prática de se conhecer e saber manusear esse recurso adaptados, compreendendo que existe a necessidade do professor conhecer esses instrumentos por conta das novas configurações que se apresentam na sala de aula com a inclusão.

\section{REFERÊNCIAS}

BRASIL. Ministério da Educação e do Desporto. Secretaria de Educação Fundamental. Parâmetros Curriculares Nacionais: Terceiro e Quarto Ciclos do Ensino Fundamental: Matemática ( $5^{\mathrm{a}}$ a $8^{\mathrm{a}}$ séries). Brasília: MEC/SEF. 1998.

BRANDÃO, J. $\mathrm{C}$.Antes de $\mathbf{P} \quad \mathbf{E} \quad \mathbf{B}$ escrevemos....introdução ao raciocínio lógicomatemático adaptado/ Jorge Brandão, Elisângela Magalhães, Ivanice Bastos- 1 ed.Curitiba, PR: CRV. 2014

BULGRAEN, V. C. O papel do professor e sua mediação nos processos de elaboração do 
conhecimento. Revista Conteúdo, Capivari, v.1, n.4, ago./dez. 2010

LIBÂNEO, J. C. Didática. 1. ed. São Paulo: Cortez, 1994

LORENZATO, S. (org): O laboratório de ensino de matemática na formação de professores. Campinas, SP: Autores Associados, p. 113-134. 2006.

\section{MAGalhães, E. B. A Sequência Fedathi na}

Deficiência Visual. Mestrado em Ensino da Matemática/Universidade Federal do Ceará, 2015.

SANTOS, M. J. C dos Reaprender frações por meio das oficinas pedagógicas: desafios para formação inicial. Dissertação (mestrado) Universidade Federal do Ceará- UFC 2007.

SFORNI, M. S. F. Aprendizagem conceitual e organização do ensino: contribuições da teoria da atividade. Araraquara: Junqueira \& Marin, 2003.

Aprendizagem conceitual e
organização de ensino: contribuições da teoria da atividade. Araraquara: JM Editora, 2004.

Aprendizagem conceitual nas séries iniciais do ensino fundamental. Educar, Curitiba, n. 28, p. 217-229, 2006. Editora UFPR 2006.

SEQUÊNICA FEDATHI: Uma proposta para o ensino de matemática e ciências / SOUSA, Francisco Edisom Eugenio, VASCONCELOS Francisco Herbert Lima, BORGES NETO, Herminio et al. [organizadores] - Fortaleza: Edições UFC, 2013.

SOUZA, M. J. A. Sequência Fedathi: apresentação e caracterização. Fortaleza: Edições UFC, 2013.

VAN HIELE, P.M. Structure and insight: a theory of Mathematics Education. Orlando: Academic

Press.

1986 